\title{
A CONCEPÇÃO DE UM PROJETO DE OBSERVATÓRIO DE QUALIDADE DE VIDA: RELATO DE UMA EXPERIÊNCIA REALIZADA EM CAMPINAS - SP.
}

\author{
Marco Akerman* \\ William Massei** \\ Suely Cabra/** \\ Angela Broch ** \\ Agenor Cremomese ${ }^{\star *}$ \\ Terezinha Silveira Alves** \\ Marisa T.M. Frichenbruder ${ }^{\star \star}$
}

\begin{abstract}
Resumo: Partindo do pressuposto que a construção de uma Cidade Saudável exige esforços de todos seus moradores e que para que isto possa ser realizado a partir de bases científicas, os autores deste artigo apresentam a experiência do Projeto Observatório de Qualidade de Vida de Campinas com vistas à construção de um instrumento, indicador de qualidade de vida. As fases descritas foram as seguintes: mobilização intersetorial; conceituação e localização de áreas administrativas a áreas de planejametno com bases espaciais para o projeto urbanístico a ser realizado por futuras secretarias de ação regionais; construção do indicador de qualidade de vida propriamente dito a partir das bases de dados demográficos de 1991; discussões e recomendações. Tal exercício lançou bases para a consolidação de um instrumetal eficiente e factivel de monitoramento continuo de um processo intersetorial de melhoria de qualidade de vida, tal qual propõe a OMS para Cidades Saudáveis.
\end{abstract}

Palavras-chave: construção; cidade saudável; qualidade de vida

* Professor Titular de Saúde Coletiva da Faculdade de Medicina do ABC, pesquisador do CEDEC, Coordenador de Saúde CASSI-SP

** Funcionários da Polícia Militar de Campinas na gestão dos Prefeitos José Roberto Magalhães Teixeira e Edvaldo Orsi. 


\section{INTRODUÇÃO}

O projeto "Cidades Saudáveis" foi criado em 1986 pela Organização Mundial da Saúde. A proposta pressupõe desenvolvimento de ações de longo-prazo que envolvam a totalidade dos setores govemamentais, privados e comunitários das cidades envolvidas, a fim de aprimorar a qualidade de vida das pessoas que residem e trabalham nas áreas urbanas.

Dentro dessa perspectiva, a administração municipal de Campinas - gestão 93-96desenvolveu projetos e programas com a diretriz básica de proporcionar a melhoria da qualidade de vida no município. Para fortalecer seu intento, e incorporar Campinas no contexto intemacional da rede de cidades saudáveis o governo municipal estabeleceu parceria com entidades que pudessem assessorar os vários projetos desenvolvidos e que tiveram como pré-requisitos: alto grau de participação social, ecossistema estável e sustentável, economia local diversificada, necessidades básicas satisfeitas, orgulho e respeito pela herança cultural, etc.

A construção de uma "Campinas Saudável" exige esforço de todos moradores da cidade, na observação cotidiana da sua qualidade de vida. A fim de criar bases sólidas e sustentáveis para esse esforço coletivo, um grupo de trabalho coordenado pela Secretaria de Governo apresentou à sociedade o projeto "Observatório da Qualidade de Vida da Cidade de Campinas". Esse projeto busca introduzir gradualmente na cultura da gestão do município de Campinas mecanismos sistêmicos e periódicos, de acompanhamento histórico da qualidade de vida na cidade.

O presente artigo discute a experiência de construção deste "observatório".

Sugeriu-se que a estruturação do Observatório fosse feita em quatro fases: (I) construção do indicador de Qualidade de Vida de Campinas (I.Q.V.) (II) estabelecimento de uma dinâmica participativa no processo de observação da qualidade de vida, com a extensão do projeto para amplos setores da comunidade (III) criação da Rede Municipal de Observação de Qualidade de Vida (IV) implantação de mecanismos e instrumentos permanentes de monitoramento da qualidade da vida no município de Campinas.

As próximas sessões dão destaque à descrição da fase I do "observatório" uma vez que as fases II, III e IV ficaram como propostas para a gestão municipal seguinte. 


\section{O PROCESSO DE MOBILIZAÇÃO INTERSETORIAL}

Em maio de 1995, a Secretaria de Govemo da Prefeitura Municipal de Campinas e a Organização Pan-americana da Saúde (OPAS) iniciaram um processo de aglutinação de profissionais de várias secretarias do município para, numa abordagem intersetorial, elaborar um "indicador composto" de qualidade de vida para a cidade de Campinas. O que justifica essa abordagem intersetorial para fenômenos ligados à qualidade de vida de populações urbanas?

DUHL (1993) noslembra que um planejamento urbano seria mais eficaz se conseguisse aglutinar visões distintas e que através de uma série de processos de negociação pudesse acomodar as diversas expectativas.

Plenamente consciente dessas questões, autores, profissionais, agências de fomento e organizações públicas que ațuam na área de planejamento urbano, em geral, e na área da saúde, em particular, vêm desde o início da década de 80 , propondo a intersetorialidade como um dos princípios fundamentais para o enfrentamento sustentável dos problemas que acometem as populações humanas (Wema, 1994). Recentemente, o projeto "Cidades Saudáveis", da Organização Mundial de Saúde - OMS resgatou mais uma vez a necessidade da intersetorialidade e fez dessa condição fator relevante para o sucesso da proposta (DUHL, 1993, WERNA, 1995).

Entretanto, sabemos da dificuldade de implementarmos uma ação intersetorial. Um trecho de Pareto (1992) ilustra bem essa dificuldade e, mais ainda, informa o porque da necessidade de se buscar formas de superação da ótica setorial: "Na prática a maioria dos projetos e programas tem uma forte conotação setorial, refletindo a estrutura da administração urbana. Se não existir um planejamento que coordene os setores, cada um irá propor seu próprio projeto, baseado em sua visão da realidade urbana, e estabelecerá seus próprios objetivos e métodos. Assim sendo, os setores com maior poder político irão provavelmente receber maior fatia dos recursos disponiveis, enquanto os mais fracos terão que competir pelas sobras. Este processo tende a agravar as distorções e as desigualdades já existentes no espaço urbano" (PARETO, 1992).

Diante disso, sugeriu-se a abordagem intersetorial e o processo de busca de consenso para a construção do indicador de qualidade de vida de Campinas, como um exercício de prática

${ }^{1} \mathrm{O}$ indicador composto é uma medida que associa diferentes variáveis sócio-econômicas e de ambiente num indicador sintético para analisar as caracteristicas de grupos populacionais vivendo em determinadas áraes geográficas (Carstair e Morris, 1989a, 1989b, Locker, 1992). 
intersetorial, partindo-se do pressuposto que a intersetorialidade não é uma condição que ocorre automaticamente, mas que deve ser construída na prática diária.

Um membro da Secretaria de Govemo, coordenou esse processo edurante dois meses percorreu quase a totalidade das secretarias, autarquias e empresas municipais para identificar profissionais que pudessem compor um grupo de trabalho.

Após a formação desse grupo intersetorial ampliado, composto de aproximadamente 25 pessoas, de 14 secretarias, 1 autarquia e 3 sociedades de economia mista, realizaram-se quatro oficinas de trabalho, em julho e agosto de 1995.

Essas oficinas tiveram como ponto de partida a discussão de três "conceitos nucleadores" - vida, espaço urbano e transetoriedade. Essa discussão foi estimulada pelo uso de textos poesias, músicas, fotografias, recortes de jomais, relacionados direta ou indiretamente com os três conceitos nucleadores já mencionados. Um trabalho de campo também foi realizado para que os membros do grupo pudessem caracterizar o espaço urbano a partir dos elementos que o constitui. Essas duas etapas an̉nteriores desembocaram na fase de construção do "indicador composto" constituída pelas seguintes tarefas: (1) decisão da base geográfica do indicador; (2) apresentação de lista de variáveis existentes nos bancos de dados do município compatíveis, com a base geográfica decidida; (3) seleção das variáveis a serem incluídas no indicador composto.

As unidades Territoriais Básicas - UTBs - estabelecidas pelo novo Plano Diretor foram escolhidas como a base geográfica do "indicador composto", e as variáveis "renda", "saúde", "educação", "transporte", "habitação", "meio-ambiente" foram eleitas como referencias para a produção de seis indicadores. Um grupo-executivo composto por sete pessoas foi formado para dar continuidade ao processo de construção do "indicador composto".

Atividades mais urgentes, ligadas à elaboração do orçamento para o ano de 1996, impediram o grupo executivo de se reunir de forma periódica no segundo semestre de 1995. Essas reuniões foram retomadas, a partir do início de 1996, e desde então esse grupo reuniu-se periodicamente até a conclusão da Fase I - construção do I.Q.V.. Devido a esses contratempos, o mesmo conseguiu reportar-se, ao já referido grupo intersetorial ampliado apenas uma vez no decorrer do seu trabalho.

Os próximos tópicos detalham conceitos e métodos com os quais esteve envolvido o grupo-executivo desde janeiro a outubro de 1996, quando se concluiu a Fase I do Observatório de Qualidade de Vida da Cidade de Campinas. 


\section{CONHECENDO AS UNIDADES DE PLANEJAMENTO TERRITORIAL DE CAMPINAS}

Nas duas últimas décadas, Campinas vem se consolidando como pólo terciário e sede de região metropolitana. Sua base produtiva e tecnológica coloca-a em posição privilegiada, em face de outras cidades para inserir-se no atual processo econômico de globalização e restruturação econômica. Entretanto, o crescimento da cidade vem se dando de forma desordenada, produzindo diferentes realidades segundo as suas diversas regiões, as quais necessitam de políticas especificas de correção e controle que busquem um desenvolvimento equilibrado.

É neste contexto que se insere o processo de revisão e detalhamento do Plano Diretor de Campinas (Prefeitura Municipal de Campinas, 1995), que coloca como orientação fundamental para a gestão da cidade, a descentralização da administração pública e a participação da comunidade. Procurou-se adotar um eixo de trabalho que tem como referência, por um lado, a preservação do patrimônio natural e a racionalização do uso dos recursos naturais e, por outro, a qualidade e o equilibrio ambiental da cidade, definido-se macrozonas que formam um gradiente quanto à factibilidade e conveniência de urbanização no curto, médio e longo prazos.

As macrozonas foram divididas em 37 áreas de planejamento (APs), para as quais definiram-se diretrizes especificas. As APs constituem recortes espaciais delimitados em função da dinâmica de estruturação urbana e da inter-relação dos problemas localmente, identificados.

Desta forma, apresentam diferentes extensões territoriais e contingentes populacionais, que variam segundo o grau de consolidação e complexidade urbana.

A área urbana do município foi dividida em 77 unidades territoriais básicas (UTBs), que correspondem, cada qual, a um bairro ou a um conjunto de bairros. O critério desta subdivisão foi a identificação de barreiras fisicàs, tais como: sistema viário, córregos e rios que separam porções do espaço urbano que guardam grau significativo de homogeneidade. Neste sentido, foram analisadas informações relativas a niveis de renda e a padrões de ocupação. A denominação das unidades territoriais básicas procurou consagrar os nomes pelos quais a população reconhece as unidades em questão.

As APs e as UTBs serão as bases espaciais para o detalhamento da legislação urbanistica. É importante destacar que a subdivisão em 37 APs e 77 UTBs guarda correspondência não só com as macrozonas, mas também com a divisão em regiões administrativas, dado que as Secretarias de Ação Regional serão o apoio do desenvolvimento e implantação das políticas 
públicas e dos planos locais. A estrutura urbana e a organização territorial aqui apresentada, considerou como elementos presentes na problematização do desenvolvimento urbano de Campinas as questões ambientais e de estrutura em especial aquelas relativas ao sistema viário e de transportes e ao saneamento básico, a isto vão se somar outros indicadores referentes ao "Nivel de Qualidade da Vida" da população residente.

A escolha desta base (UTBS) para o índice de Qualidade de Vida consolida esta sistemática, integrada ao trabalho das SAR's e ao esforço feito no âmbito do convênio com a Organização Pan-americana de Saúde - OPAS, no "Programa Campinas Cidade Saudável", cujo objetivo principal consiste em propiciar a melhoria da qualidade de vida em Campinas para toda a população, com ênfase nas camadas mais carentes e desassistidas.

\section{METODOLOGIA DE CONSTRUÇÃO DO "INDICADOR DE QUALIDADE DE VIDA"}

\section{Objetivos}

Os seguintes objetivos permearam a construção do indicador de qualidade de vida de Campinas:

\section{introduzir a concepção intersetorial da qualidade de vida}

olhar para a cidade sob a ótica de unidades territoriais distintas, examinando as limitações e potenciais das fontes de dados municipais; qualidade de vida;

elaborar um modelo técnico para a construção de um indicador composto de introduzir a discussão sobre definição de parâmetros mínimos de qualidade de vida;

problematizar conceitos relacionados ao tema da qualidade de vida;

Após definição, pelo grupo intersetorial ampliado, das variáveis-referência - "renda", "saúde", "educação" , "transporte", "habitação", "meio - ambiente" - e das UTBs como base geográfica do indicador composto, iniciou-se o processo técnico e elaboração do Indicador de Qualidade de Vida. Este processo teve a duração de 10 meses (janeiro a outubro) e foi conduzido 
por um grupo-executivo composto por sete pessoas. Otrabalho de computação e compatibilização dos dados foi desenvolvido portécnica da Secretaria de Govemo com um profundo conhecimento dos bairros e ruas de Campinas.

\section{Passos seguidos para a construção do indicador}

1. A partir das variáveis referências - "renda", "saúde", "educação", "transporte", "habitação", "meio-ambiente" - os seguinte indicadores foram selecionados: (1) percentual de chefes de família sem rendimento ou com até 2 salários mínimos; (2) percentual de população analfabeta de 15 anos ou mais; (3) percentual de domicílios em aglomerados sub-normais; (4) percentual de domicílios com acesso precário à água; (5) percentual de domicílios com acesso precário ao esgoto; (6) percentual de domícílios com acesso precário à coleta de lixo.

2. Tabulação dos valores dos indicadores acima para cada UTB, a partir dos dados do Censo IBGE, 1991 após exclusão para fins de análise das seguintes áreas: UTBs com população igual a zero, UTBs com população inferior a $0,1 \%$ da população total da cidade e a zona rural das áreas de Planejamento. Este exercício resultou na exclusão de 17 UTBs e áreas rurais representando apenas $2 \%$ da população total da cidade. Para as UTBs $(21,22,22-B, 23,38,42)$ inexistiam algumas variáveis, nesse caso admitiu-se o valor zero para os respectivos indicadores.

3. Estabelecimento de dois critérios de relação - "absoluto" e "relativo" - entre os dados de cada UTB e a cidade como um todo. A relação "absoluta" levou em conta, para cada indicador, a representação percentual do valor da UTB em relação ao valor total da cidade. Por exemplo, a UTB 47, possui 4099 chefes de família sem rendimento ou com até 2 salários mínimos que representam $1,95 \%$ de todos os domicílios de chefes de família sem rendimento ou com até 2 salários-mínimos da cidade de Campinas. A relação "relativa", por outro lado, apontou a relação intra-UTB para cada indicador. Por exemplo, tomando novamente o exemplo da renda para a UTB 47, os 4099 domicílios de chefes de família sem rendimento ou com até 2 salários mínimos representam $29,21 \%$ e todos os domicílios totais existentes na UTB 47.

Se em termos absolutos, a UTB 44 é a que apresenta o maior percentual de "pobres" da cidade, em termos relativos, o seu número de "pobres', como uma proporção do total dos domicílio da UTB, é de $26,58 \%$, abaixo da proporção de outras UTBs como a 8 com $37,89 \%$ e a $48 \mathrm{com}$ $33,9 \%$ de "pobres" em relação aos "não pobres", dentro da UTB.

Em se adotando o critério "absoluto", políticas públicas poderiam priorizar ações dirigidas 
à UTB 47, enquanto, ao se levar em conta o critério "relativo" as UTBs 8 e 48 passariam a ser mais prioritánias.

Quais as principais diferenças conceituais entre o estabelecimento dos dois critérios de relação: "absoluto" e "relativo"?

O primeiro indica qual é a proporção na UTB em relação a cidade como um todo, indicando um ranking de magnitudes quantitativas entre as UTBs. A maior quantidade de problemas ficaria no topo das prioridades, onde provavelmente o custo relativo de atender as famílias naquela UTB seria menor que naquelas UTBs, com menor quantidade de problemas, além de que, $\mathrm{o}$ atendimento de maiores quantidades absolutas garante maior visibilidade ao programa.

O critério "relativo" por outro lado, não releva tanto as quantidades absolutas de problemas mas a relação das propọç̧̃es entre "domicílios com problemas" e "domicílios sem problemas" numa mesma UTB. Aqui, o foco é no local e não no âmbito geral da cidade. Enquanto o critério "absoluto" dá suporte ao macroplanejamento urbano de caráter mais centralizado, o critério "relativo" poderia favorecer um planejamento mais local e descentralizado. A tabela 1 apresenta as 60 UTBs e seus respectivos percentuais "absoluto" e "relativo" em relação aos chefes e família s/ renda ou com até 2 salários mínimos.

TABELA 1: Distribuição dos percentuais "relativo" e "absoluto" para as 60 UTBS em relação aos chefes de familia sem renda ou com até 2 salários mínimos

\begin{tabular}{|c|c|c|c|c|c|c|}
\hline \multirow[b]{2}{*}{ BAIRRO } & \multirow{2}{*}{$\begin{array}{c}\mathrm{N}^{0} \text { Chefes } \\
\text { de } \\
\text { Familia }\end{array}$} & \multirow{2}{*}{$\begin{array}{c}\text { Chefes } \\
\text { Família s/ } \\
\text { renda ou até } 2 \\
\text { sal.min }\end{array}$} & \multicolumn{2}{|c|}{ RELATIVO } & \multicolumn{2}{|c|}{ ABSOLUTO } \\
\hline & & & Ranking & $\% \mathrm{~s} /$ UTB & Ranking & $\%$ s/ UTB \\
\hline Guará & 948 & 182 & 0,25 & 19,20 & 0,79 & 0,09 \\
\hline Centro Br. Geraldo & 2843 & 566 & 0,22 & 19,91 & 0,33 & 0,27 \\
\hline Cidade Universitária & 1934 & 91 & 0,91 & 4,96 & 0,90 & 0,04 \\
\hline Real Parque & 799 & 228 & $-0,11$ & 28,54 & 0,73 & 0,11 \\
\hline $\begin{array}{l}\text { PUCC/ Pq das } \\
\text { Universidades }\end{array}$ & 227 & 86 & $-0,43$ & 37,89 & 0,90 & 0,04 \\
\hline S. Martin & 544 & 151 & $-0,08$ & 27,76 & 0,83 & 0,07 \\
\hline São Marcos/ Amarais & 4344 & 2093 & $-0,79$ & 48,18 & $-0,34$ & 1,00 \\
\hline $\begin{array}{l}\text { NV Aparecida/ } \\
\text { Pe Anchieta }\end{array}$ & 4551 & 1297 & $-0,11$ & 28,5 & $-0,08$ & 0,62 \\
\hline $\begin{array}{l}\text { Fazendinha/ Sta } \\
\text { Bárbara }\end{array}$ & 2992 & 820 & $-0,07$ & 27,41 & 0,02 & 0,39 \\
\hline Pq Via Norte & 2378 & 768 & $-0,24$ & 32,2 & 0,08 & 0,37 \\
\hline
\end{tabular}




\begin{tabular}{|c|c|c|c|c|c|c|c|}
\hline \multirow[b]{2}{*}{ UTB } & \multirow[b]{2}{*}{ BAIRRO } & \multirow[b]{2}{*}{$\begin{array}{c}N^{0} \text { Chefes } \\
\text { de } \\
\text { Familia }\end{array}$} & \multirow{2}{*}{$\begin{array}{c}\text { Chefes } \\
\text { Familia s/ } \\
\text { renda ou até } 2 \\
\text { sal.min }\end{array}$} & \multicolumn{2}{|c|}{ RELATIVO } & \multicolumn{2}{|c|}{ ABSOLUTO } \\
\hline & & & & Ranking & $\% s /$ UTB & Ranking & $\%$ s/ UTB \\
\hline 14 & Faz. Chapadão & 195 & 6 & 1,00 & 3,08 & 1,00 & 0,00 \\
\hline 16 & VL Nova & 1738 & 254 & 0,46 & 14,61 & 0,00 & 0,12 \\
\hline 17 & Chapadão & 4262 & 1149 & $-0,05$ & 26,96 & $-0,03$ & 0,55 \\
\hline 18 & Castelo & 3351 & 374 & 0,62 & 11,16 & 0,56 & 0,18 \\
\hline 19 & Bonfim & 2608 & 564 & 0,14 & 21,63 & 0,33 & 0,27 \\
\hline $\begin{array}{l}20 \\
21\end{array}$ & $\begin{array}{l}\text { Jd Aurélia } \\
\text { C.Gomes/Monte Belo/ }\end{array}$ & 6800 & 1132 & 0,37 & 16,65 & $-0,03$ & 0,54 \\
\hline 22 & $\begin{array}{l}\text { Chac. Garc } \\
\text { Jd. Miriam/ Pq }\end{array}$ & 253 & 137 & $-1,00$ & 54,15 & 0,84 & 0,07 \\
\hline 23 & $\begin{array}{l}\text { Xangrilá } \\
\text { VL Costa e Silva / VL }\end{array}$ & 328 & 86 & $-0,03$ & 26,22 & 0,90 & 0,04 \\
\hline 24 & $\begin{array}{l}\text { M.V.Cury } \\
\text { Monsões Sto Antonio/ }\end{array}$ & 3838 & 1063 & $-0,08$ & 27,7 & $-0,01$ & 0,51 \\
\hline 25 & $\begin{array}{l}\text { Sta Cândida } \\
\text { Primavera/Pq }\end{array}$ & 774 & 178 & 0,07 & 23,00 & 0,79 & 0,08 \\
\hline & Taquaral & 2135 & 417 & 0,23 & 19,53 & 0,51 & 0,20 \\
\hline 26 & São Quirino & 5454 & 1710 & $-0,21$ & 31,35 & $-0,22$ & 0,81 \\
\hline 27 & Jd. N Sra. Auxiliadora & 6354 & 1145 & 0,3 & 18,02 & $-0,03$ & 0,55 \\
\hline $\begin{array}{l}28 \\
29\end{array}$ & $\begin{array}{l}\text { Pq. Brasilia } \\
\text { Carrefour/ Galeria/ }\end{array}$ & 3351 & 1045 & $-0,2$ & 31,18 & 0,00 & 0,50 \\
\hline & Feac & 455 & 186 & $-0,54$ & 40,88 & 0,78 & 0,09 \\
\hline 30 & Guanabara & 4360 & 474 & 0,64 & 10,87 & 0,44 & 0,23 \\
\hline 31 & Cambui & 9126 & 768 & 0,75 & 8,42 & 0,08 & 0,37 \\
\hline 32 & Flamboyant & 4607 & 643 & 0,49 & 13,96 & 0,23 & 0,31 \\
\hline 33 & VI. Brandina & 1392 & 309 & 0,11 & 22,20 & 0,64 & 0,15 \\
\hline 34 & Centro Br. Geraldo & 6886 & 687 & 0,68 & 9,98 & 0,18 & 0,33 \\
\hline 35 & Bosque & 5408 & 619 & 0,61 & 11,45 & 0,26 & 0,29 \\
\hline 36 & N.Campinas & 1778 & 128 & 0,81 & 7,2 & 0,85 & 0,06 \\
\hline 38 & Notre Dame/Alto Nova & & & & & & \\
\hline & Campinas & 313 & 48 & 0,43 & 15,34 & 0,95 & 0,02 \\
\hline 39 & São Conrado & 235 & 33 & 0,49 & 14,04 & 0,97 & 0,02 \\
\hline 40 & Centro Souzas & 2088 & 612 & $-0,13$ & 29,31 & 0,27 & 0,29 \\
\hline $\begin{array}{l}42 \\
44\end{array}$ & $\begin{array}{l}\text { Joaquim Egidio } \\
\text { Jd. Garcia / Campos }\end{array}$ & 416 & 163 & $-0,48$ & 39,18 & 0,81 & 0,08 \\
\hline & Eliseos & 15521 & 4125 & $-0,04$ & 26,58 & $(1,00)$ & 1,96 \\
\hline $\begin{array}{l}45 \\
46\end{array}$ & $\begin{array}{l}\text { Pq. Valência } \\
\text { Campo Grande/ }\end{array}$ & 6159 & 2242 & $-0,38$ & 36,4 & $-0,39$ & 1,07 \\
\hline & Florence & 4227 & 1679 & 0,50 & 39,72 & $-0,21$ & 0,80 \\
\hline 47 & NV.Campos Eliseos/ & & & & & & \\
\hline & Sta Lucia & 14031 & 4099 & $-0,13$ & 29,21 & $-0,99$ & 1,95 \\
\hline 48 & Mauro Marcondes/O & & & & & & \\
\hline & Verde & 925 & 3136 & $-0,29$ & 33,9 & $-0,68$ & 1,49 \\
\hline 49 & Maria Rosa & 1913 & 569 & $-0,15$ & 29,74 & 0,32 & 0,27 \\
\hline $\begin{array}{l}50 \\
55\end{array}$ & Jd. S. Cristovão & 2453 & 813 & $-0,27$ & 33,14 & 0,03 & 0,39 \\
\hline & $\mathrm{Pq}$ Industrial & 11048 & 2520 & 0,08 & 22,81 & $-0,48$ & 1,20 \\
\hline 56 & Ponte Preta & 4133 & 837 & 0,20 & 20,25 & 0,00 & 0,40 \\
\hline $\begin{array}{l}57 \\
58\end{array}$ & $\begin{array}{l}\text { Jd. Proença } \\
\text { S. Fernando/NL }\end{array}$ & 5847 & 1199 & 0,19 & 20,51 & $-0,05$ & 0,57 \\
\hline & Orosimbo Maia/C & 4974 & 1705 & $-0,31$ & 34,28 & $-0,21$ & 0,81 \\
\hline 59 & V.Pompéia/Jd do & & & & & & \\
\hline 60 & $\begin{array}{l}\text { Lago } \\
\text { NV Eurona/Pa da }\end{array}$ & 5917 & 1453 & 0,00 & 24,56 & $-0,13$ & 0,69 \\
\hline & Figueira & 5570 & 1418 & 0,00 & 25,46 & $-0,12$ & 0,68 \\
\hline
\end{tabular}




\begin{tabular}{|c|c|c|c|c|c|c|c|}
\hline \multirow[b]{2}{*}{ UTB } & \multirow[b]{2}{*}{ BAIRRO } & \multirow[b]{2}{*}{$\begin{array}{c}N^{0} \text { Chefes } \\
\text { de } \\
\text { Familia }\end{array}$} & \multirow{2}{*}{$\begin{array}{c}\text { Chefes } \\
\text { Familia s/ } \\
\text { renda ou até } 2 \\
\text { sal.min }\end{array}$} & \multicolumn{2}{|c|}{ RELATIVO } & \multicolumn{2}{|c|}{ ABSOLUTO } \\
\hline & & & & Ranking & $\%$ s/ UTB & Ranking & $\% s /$ UTB \\
\hline 6 & $\begin{array}{l}\text { Jd. Dos Oliveiras/ } \\
\text { wift }\end{array}$ & 9049 & 2324 & $-0,01$ & 25,68 & $-0,42$ & 1,11 \\
\hline 62 & Jd.Esmeraldina/Jd.S. & & & & & & \\
\hline & Pedro/Jd.S.V & 5401 & 1662 & $-0,19$ & 30,77 & $-0,2$ & 0,79 \\
\hline $\begin{array}{l}63 \\
64\end{array}$ & $\begin{array}{l}\mathrm{Pq} \text { Jambeiro/Remonta } \\
\text { Icarai/Jd das }\end{array}$ & 393 & 121 & $-0,19$ & 30,79 & 0,86 & 0,06 \\
\hline & Bandeiras & 2951 & 1109 & $-0,42$ & 37,58 & $-0,02$ & 0,53 \\
\hline $\begin{array}{l}65 \\
66\end{array}$ & $\begin{array}{l}\text { NV Mercedes } \\
\text { Jd São Domingos/CP }\end{array}$ & 306 & 138 & $-0,68$ & 45,1 & 0,84 & 0,07 \\
\hline & Belo & 1238 & 447 & $-0,37$ & 36,11 & 0,47 & 0,21 \\
\hline 67 & Jd Fernanda & 455 & 75 & 0,38 & 16,48 & 0,92 & 0,04 \\
\hline 22-B & $\mathrm{Pq}$ Imperador & 304 & 101 & $-0,27$ & 33,22 & 0,89 & 0,05 \\
\hline 38-A & Bairro das Palmeiras & 350 & 53 & 0,44 & 15,14 & 0,94 & 0,03 \\
\hline $66-A$ & JD. NV América & 672 & 223 & $-0,27$ & 33,18 & 0,74 & 0,11 \\
\hline TOTA & & 218760 & 53754 & 3,94 & 1525,86 & 16,19 & 25,65 \\
\hline
\end{tabular}

FONTE: Observatório de Qualidade de Vida de Campinas, 1996.

\section{Em seguida procedeu-se à construção propriamente dita do indicador de qualidade} de vida que incluiu as seguintes etapas:

. Classificação em ordem decrescente dos percentuais "absolutos" e "relativos" obtidos para as 60 UTBs em relação aos indicadores selecionados.

. Cálculo da média dos percentuais "absolutos" e "relativos" para todos os indicadores selecionados.

Em média estabelece-se como um limiar para cada indicador. Esse limiar foi ocnsiderado o "padrão mínimo" aceitável para cada indicador. Para os percentuais "absolutos" e "relativos" baixo do padrão mínimo" atribuíram-se índices negativos entre 0 e 1 para percentuais "absolutos" e "relativos" acima do "padrão mínimo" atribuíram-se índices positivos entre $0 \mathrm{e}+1^{2}$. A adoção de padrões baseados na média da cidade, e não em padrões nacionais ou intemacionais já consagrados, subentende um processo de melhoria contínua em que a cidade se compara sempre consigo mesma, uma vez que esses limiares se modificam ao longo do tempo ${ }^{3}$.

\footnotetext{
2 Os valores entre 0 e 1 e -1 são calculados a partir das fórmulas: Zijt $=[X i j t-\operatorname{minXijt}]$ onde $X$ representa as variáveis consideráveis [máx Xijt - min Xijt] (PUND; 1993).

${ }^{3}$ Essa metodologia está baseada na elaboração do indice de desenvolvimento humano (idh) da ONU/PNUD e na construção do indicador composto de exclusão/inclusão social da cidade de São Paulo da PUC/CEDEC/Comissão Arquideocesana da Campanha da Fraternidade (Sposati et al, 1996)
} 
TABELA 2: Distribuição dos indices de infraestrutura para as 60 UTBs de Campinas

\begin{tabular}{|c|c|c|c|c|c|c|c|c|}
\hline UTB & NOME & ÁGUA & água & ESG. & esgoto & LIXO & lixo & $\begin{array}{l}\text { SOMA DE INFRA- } \\
\text { ESTRUTURA }\end{array}$ \\
\hline 2 & Guará & $-0,04$ & $-0,01$ & $-0,05$ & $-0,03$ & 0,00 & 0,00 & $-0,03$ \\
\hline 4 & Centro BR.Geraldo & 0,84 & 0,17 & 0,65 & 0,32 & 0,85 & 0,25 & 0,75 \\
\hline 5 & Cidade Universitária & 0,95 & 0,19 & 0,91 & 0,46 & 0,95 & 0,28 & 0,93 \\
\hline 7 & Real Parque & 0,44 & 0,09 & $-0,89$ & $-0,45$ & 0,29 & 0,09 & $-0,27$ \\
\hline 8 & PUCC/Pq. das & & & & & & & \\
\hline & Universidades & $-0,11$ & $-0,02$ & 0,33 & 0,16 & $-0,07$ & $-0,02$ & 0,12 \\
\hline 9 & S.Martin & $-0,13$ & $-0,03$ & 0,00 & 0,00 & $-0,16$ & $-0,05$ & $-0,07$ \\
\hline $\begin{array}{l}10 \\
11\end{array}$ & $\begin{array}{l}\text { São Marcos/Amarais } \\
\text { NV Aparecida/Pe }\end{array}$ & 0,00 & 0,00 & $-0,10$ & $-0,05$ & $-0,14$ & $-0,04$ & $-0,09$ \\
\hline 12 & $\begin{array}{l}\text { Anchieta } \\
\text { Fazendinha/Sta }\end{array}$ & 0,26 & 0,05 & 0,43 & 0,22 & 0,88 & 0,26 & 0,53 \\
\hline & Bárbara & 0,00 & 0,00 & $-0,17$ & $-0,09$ & 0,77 & 0,23 & 0,14 \\
\hline 13 & Pq Via Norte & 0,96 & 0,19 & 0,95 & 0,48 & 0,92 & 0,28 & 0,94 \\
\hline 14 & Faz. Chapadão & 0,91 & 0,18 & 1,00 & 0,50 & 0,81 & 0,24 & 0,92 \\
\hline 15 & Faz. Sta. Elisa & 0,07 & 0,01 & 1,00 & 0,50 & 0,55 & 0,17 & 0,68 \\
\hline 16 & VL Nova & 1,00 & 0,20 & 0,99 & 0,50 & 0,55 & 0,17 & 0,68 \\
\hline 17 & Chapadão & 0,97 & 0,19 & 0,77 & 0,39 & 0,93 & 0,28 & 0,86 \\
\hline 18 & Castelo & 0,99 & 0,20 & 0,98 & 0,49 & 0,98 & 0,29 & 0,98 \\
\hline 19 & Bonfim & 1,00 & 0,20 & 0,98 & 0,49 & 0,98 & 0,29 & 0,98 \\
\hline 20 & Jd. Aurélia & 0,99 & 0,20 & 0,97 & 0,48 & 0,92 & 0,28 & 0,96 \\
\hline 21 & $\begin{array}{l}\text { C.Gomes/Monte Belo/t } \\
\text { Chac. Ga } \\
\text { Jd. Miriam/Pq }\end{array}$ & 0,84 & $-0,17$ & $-0,65$ & $-0,32$ & $-1,00$ & $-0,30$ & $-0,79$ \\
\hline & Xangrilá & $-0,14$ & $-0,03$ & $-1,00$ & $-0,50$ & $-0,24$ & $-0,07$ & $-0,60$ \\
\hline 23 & VL Costa e Silva/ & & & & & & & \\
\hline & VL M.V.Cury & 0,93 & 0,19 & $-0,48$ & $-0,24$ & 0,94 & 0,28 & 0,23 \\
\hline 24 & Monsōes Sto Antônio/ & & & & & & & \\
\hline 25 & $\begin{array}{l}\text { Sta Cândida } \\
\text { Primavera/Pq }\end{array}$ & 0,95 & 0,19 & $-0,48$ & $-0,24$ & 0,94 & 0,28 & 0,23 \\
\hline & Taquaral & 0,97 & 0,19 & 0,97 & 0,48 & 0,95 & 0,28 & 0,96 \\
\hline $\begin{array}{l}26 \\
27\end{array}$ & $\begin{array}{l}\text { São Quirino } \\
\text { Jd. N.Sra. }\end{array}$ & 0,79 & 0,16 & 0,43 & 0,22 & 0,42 & 0,13 & 0,50 \\
\hline & Auxiliadora & 1,00 & 0,20 & 0,99 & 0,49 & 0,99 & 0,3 & 0,48 \\
\hline $\begin{array}{l}28 \\
29\end{array}$ & $\begin{array}{l}\text { Pq. Brasilia } \\
\text { Carrefour/Galeria/ }\end{array}$ & 0,74 & $-0,02$ & $-0,12$ & $-0,06$ & $-0,3$ & $-0,09$ & $-0,17$ \\
\hline & FEAC & $-0,12$ & $-0,02$ & $-0,12$ & $-0,06$ & $-0,30$ & $-0,09$ & $-0,17$ \\
\hline 30 & Guanabara & 1,00 & 0,20 & 0,99 & 0,50 & 1,00 & 0,30 & 0,99 \\
\hline 31 & Cambui & 1,00 & 0,20 & 0,99 & 0,50 & 0,99 & 0,30 & 0,99 \\
\hline 32 & Flamboyant & 0,90 & 0,18 & 0,61 & 0,30 & 0,73 & 0,22 & 0,70 \\
\hline 33 & VL. Brandina & 0,56 & 0,11 & 0,00 & 0,00 & 0,18 & 0,05 & 0,16 \\
\hline 34 & Centro & 1,00 & 0,20 & 1,00 & 0,50 & 1,00 & 0,30 & 1,00 \\
\hline 35 & Bosque & 0,99 & 0,20 & 0,98 & 0,49 & 1,00 & 0,30 & 0,99 \\
\hline 36 & N.Campinas & 0,99 & 0,20 & 0,95 & 0,47 & 0,97 & 0,29 & 0,96 \\
\hline 38 & Nova Campinas & 0,42 & 0,08 & 0,84 & 0,42 & $-0,07$ & $-0,02$ & 0,48 \\
\hline 39 & São Conrado & 0,81 & 0,16 & $-0,47$ & $-0,23$ & 0,00 & 0,00 & $-0,07$ \\
\hline 40 & Centro Souzas & 0,28 & 0,06 & 0,24 & 0,12 & 0,73 & 0,22 & 0,40 \\
\hline 42 & Joaquim Egidio & $-0,21$ & $-0,04$ & $-0,11$ & $-0,05$ & $-0,27$ & $-0,08$ & $-0,18$ \\
\hline 44 & Jd. Garcia/Campos & & & 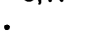 & & & & \\
\hline & Eliseos & 0,93 & 0,19 & 0,79 & 0,40 & 0,59 & 0,18 & 0,76 \\
\hline $\begin{array}{l}45 \\
46\end{array}$ & $\begin{array}{l}\text { Pq Valência } \\
\text { Campo Grande/ }\end{array}$ & 0,54 & 0,11 & $-0,18$ & $-0,09$ & 0,04 & 0,01 & 0,03 \\
\hline & Florence & $-0,14$ & $-0,03$ & $-0,18$ & $-0,09$ & $-0,17$ & $-0,05$ & $-0,17$ \\
\hline 47 & $\begin{array}{l}\text { NV.Campos Eliseos/ } \\
\text { Sta Lúcia }\end{array}$ & 0,54 & 0,11 & 0,34 & 0,17 & 0,13 & 0,04 & 0,32 \\
\hline
\end{tabular}




\begin{tabular}{|c|c|c|c|c|c|c|c|c|}
\hline UTB & NOME & ÁGUA & água & ESG. & esgoto & LIXO & lixo & $\begin{array}{l}\text { SOMA DE INFRA- } \\
\text { ESTRUTURA }\end{array}$ \\
\hline 48 & $\begin{array}{l}\text { Mauro Marcondes/ } \\
\text { O Verde }\end{array}$ & 0,37 & 0,07 & 0,19 & 0,09 & 0,16 & 0,05 & 0,21 \\
\hline 49 & Maria Rosa & 0,43 & 0,09 & 0,42 & 0,21 & 0,09 & 0,03 & 0,33 \\
\hline 50 & Jd. S. Cristovão & 0,02 & 0,00 & $-0,83$ & $-0,41$ & $-0,07$ & $-0,02$ & $-0,44$ \\
\hline $\begin{array}{l}51 \\
55\end{array}$ & $\begin{array}{l}\text { DIC's/COHAB } \\
\text { VL Teixeira/Pq Itália/ }\end{array}$ & 0,85 & 0,17 & 0,89 & 0,44 & 0,81 & 0,24 & 0,85 \\
\hline & $\mathrm{Pq}$ Indus & 0,99 & 0,20 & 0,98 & 0,49 & 0,97 & 0,29 & 0,98 \\
\hline 56 & Ponte Preta & 1,00 & 0,20 & 0,99 & 0,49 & 0,98 & 0,29 & 0,99 \\
\hline $\begin{array}{l}57 \\
58\end{array}$ & $\begin{array}{l}\text { JS Proença } \\
\text { S.Fernando NL }\end{array}$ & 0,97 & 0,19 & 0,75 & 0,37 & 0,88 & 0,26 & 0,83 \\
\hline 59 & $\begin{array}{l}\text { Orosimbo Maia } \\
\text { V.Pompéia/Jd do }\end{array}$ & 0,42 & 0,08 & 0,01 & 0,00 & 0,21 & 0,06 & 0,15 \\
\hline 60 & $\begin{array}{l}\text { Lago } \\
\text { NV Europa/Pq da }\end{array}$ & 0,99 & 0,20 & 0,83 & 0,42 & 0,83 & 0,25 & 0,86 \\
\hline 61 & $\begin{array}{l}\text { Figuera } \\
\text { Jd. Dos Oliveiras / }\end{array}$ & 0,94 & 0,19 & 0,91 & 0,46 & 0,79 & 0,24 & 0,88 \\
\hline 62 & $\begin{array}{l}\text { SWIFT } \\
\text { Jd. Esmeraldina/Jd }\end{array}$ & 0,95 & 0,19 & 0,70 & 0,35 & 0,71 & 0,21 & 0,75 \\
\hline 63 & $\begin{array}{l}\text { S.Pedro/Jd } \\
\text { Pq Jambeiro/ }\end{array}$ & 0,82 & 0,16 & 0,21 & 0,10 & 0,23 & 0,07 & 0,34 \\
\hline 64 & $\begin{array}{l}\text { Remonta } \\
\text { lcarai/Jd das }\end{array}$ & 0,24 & 0,05 & $-0,88$ & $-0,44$ & $-0,02$ & $-0,01$ & $-0,40$ \\
\hline & Bandeiras & 0,19 & 0,04 & $-0,15$ & $-0,07$ & 0,02 & 0,01 & $-0,03$ \\
\hline $\begin{array}{l}65 \\
66\end{array}$ & $\begin{array}{l}\text { NV Mercedes } \\
\text { Jd. São Domingos/ }\end{array}$ & $-0,13$ & $-0,03$ & 0,00 & 0,00 & $-0,16$ & $-0,05$ & $-0,08$ \\
\hline & CP Belo & $-1,00$ & $-0,2$ & $-0,47$ & $-0,23$ & $-0,37$ & $-0,11$ & $-0,54$ \\
\hline 67 & Jd. Fernanda & $-0,24$ & $-0,05$ & 0,17 & 0,08 & $-0,29$ & $-0,09$ & $-0,05$ \\
\hline $\begin{array}{l}22-B \\
38-A\end{array}$ & $\begin{array}{l}\text { Pq. Imperador } \\
\text { Bairro das }\end{array}$ & $-0,32$ & $-0,06$ & $-0,66$ & $-0,33$ & $-0,05$ & $-0,02$ & $-0,41$ \\
\hline & Palmeiras & 0,68 & 0,14 & 0,27 & 0,13 & $-0,04$ & $-0,01$ & 0,26 \\
\hline $66-A$ & $\begin{array}{c}\text { Jd. Nova América } \\
\text { A }\end{array}$ & $\begin{array}{l}-0,19 \\
B\end{array}$ & $\begin{array}{l}-0,04 \\
C \\
A^{\star} 0,2\end{array}$ & $\begin{array}{l}-0,93 \\
D\end{array}$ & $\begin{array}{l}-0,46 \\
E \\
C^{\star} 0,5\end{array}$ & $\begin{array}{l}-0,71 \\
F\end{array}$ & $\begin{array}{l}0,21 \\
G \\
E^{\star} 0,3\end{array}$ & $-0,29$ \\
\hline
\end{tabular}

Fonte: Observatório de Qualidade de Vida de Campinas, 1996.

O I.Q.V. de cada UTB foi formado então a partir da soma dos índices obtidos em relação às respectivas variáveis renda, educação, habitação e infra- estrutura sanitária.

As tabelas 3 e 4 mostram a posição das UTBs no ranking dos I.Q.V. "absoluto" e "relativo". Para uma melhor compreensão a variação inicial entre -1 e +1 foi transformada proporcionalmente entre +10 e -10 significando o grau de maior ou menor afastamento do "padrão mínimo". Para os valores "absolutos" há 13 UTBs com I.Q.V. negativos e para os valores "relativos" os I.Q.V. negativos representam 18 UTBs. 
Os índices obtidos em relação aos indicadores relacionados com a água, esgoto e lixo para cada UTB foram multiplicados, respectivamente, por $0,2,0,3$ e 0,5 atribuindoIhes peso (importância) diferenciado para conformação do indicador de "infra-estrutura sanitária" conforme representado Tabela 2.

TABELA 3: Posição das UTBs no ranking dos IQV "absoluto"

\begin{tabular}{|c|c|c|c|c|}
\hline RANKING & BAIRRO & UTB SAR & POP & IQV \\
\hline $1^{\circ}$ & Cidade Universitária & $5 \mathrm{~N}$ & 7.469 & 9,58 \\
\hline $2^{3}$ & Faz. Chapadão & $14 \mathrm{~N}$ & 876 & 9,35 \\
\hline $3^{3}$ & Cambui & $31 \mathrm{~L}$ & 26.168 & 9,31 \\
\hline $4^{3}$ & N.Campinas & $36 \mathrm{~L}$ & 7.072 & 9,2 \\
\hline $5^{3}$ & Centro & $34 \mathrm{~L}$ & 16.626 & 9,18 \\
\hline $6^{3}$ & Bosque & $35 \mathrm{~L}$ & 14.645 & 8,99 \\
\hline $7^{\circ}$ & Castelo & $18 N$ & 12.114 & 8,88 \\
\hline $8^{3}$ & Guanabara & $30 \mathrm{~L}$ & 11.560 & 8,85 \\
\hline $9^{3}$ & VL Nova & $16 \mathrm{~N}$ & 6.010 & 7,77 \\
\hline $10^{2}$ & Jd. N.Sra. Auxiliadora & $27 \mathrm{~L}$ & 22.197 & 7,41 \\
\hline $11^{\circ}$ & Ponte Prela & $56 \mathrm{~S}$ & 14.075 & 7,16 \\
\hline $12^{3}$ & Jd. Aurélia & $20 \mathrm{~N}$ & 25.305 & 6,99 \\
\hline $13^{\circ}$ & Bonfim & $19 N$ & 8.623 & 6,79 \\
\hline $14^{\circ}$ & VL Teixeira/Pq Itália/Pq Industrial & $55 \mathrm{~N}$ & 38.782 & 6,5 \\
\hline 15 & Primavera/Pq Taquaral & $25 \mathrm{~N}$ & 8.539 & 6,45 \\
\hline 16' & Notre Dame/Alto Nova Campinas & $38 \mathrm{~L}$ & 1.251 & 5,97 \\
\hline $17^{\circ}$ & Flamboyant & $32 \mathrm{~L}$ & 16.730 & 5,66 \\
\hline $18^{\circ}$ & Centro BR Geraldo & $4 \mathrm{~N}$ & 10.831 & 5,51 \\
\hline $19^{3}$ & VL Costa e SilvaNL M.V. Cury & $23 \mathrm{~L}$ & 15.714 & 5,19 \\
\hline $20^{3}$ & DIC'S/COHAB & 510 & 26.989 & 5,04 \\
\hline $21^{\circ}$ & NV Europa/Pq da Figueira & $60 \mathrm{~S}$ & 21.544 & 4,88 \\
\hline $22^{3}$ & Pq. Via Norte & $13 \mathrm{~N}$ & 9.458 & 4,57 \\
\hline $23^{3}$ & V.Pompéia/Jd do Lago & $59 \mathrm{~S}$ & 21.995 & 4,19 \\
\hline $24^{\circ}$ & Jd. Proença & $57 \mathrm{~S}$ & 21.268 & 3,86 \\
\hline 25 & Monsões Sto Antonio/Sta Cândida & $24 \mathrm{~L}$ & 3.285 & 3,44 \\
\hline $26^{\circ}$ & Guará & $2 \mathrm{~N}$ & 3.744 & 3,32 \\
\hline $27^{\circ}$ & Chapadão & $17 \mathrm{~N}$ & 16.462 & 3,22 \\
\hline $28^{\circ}$ & Jd. Fernanda & $67 \mathrm{~S}$ & 2.143 & 2,88 \\
\hline $29^{\circ}$ & NV Aparecida/Pe Anchieta & $11 \mathrm{~N}$ & 18.597 & 2,65 \\
\hline $30^{3}$ & Bairro das Palmeiras & 38-A L & 1.520 & 2,41 \\
\hline $31^{\circ}$ & Jd. dos Oliveiras/Swift & $61 \mathrm{~S}$ & 33.682 & 2,24 \\
\hline $32^{\circ}$ & Jd. Garcia/Campos Eliseos & 440 & 59.354 & 1,8 \\
\hline $33^{3}$ & Maria Rosa & 490 & 59.354 & 1,8 \\
\hline $34^{3}$ & São Conrado & $39 \mathrm{~L}$ & 1.029 & 1,26 \\
\hline $35^{\circ}$ & PUCC/Pq das Universidades & $8 \mathrm{~N}$ & 958 & 1,23 \\
\hline $36^{3}$ & Pq. Jambeiro/Remonta & $63 \mathrm{~S}$ & 1.613 & 1 \\
\hline $37^{\circ}$ & S. Martin & $9 \mathrm{~N}$ & 2.380 & 0,78 \\
\hline $38^{3}$ & Pq Imperador & 22-B L & 1.289 & 0,67 \\
\hline $39^{\circ}$ & Centro Souzas & $40 \mathrm{~L}$ & 8.219 & 0,66 \\
\hline $40^{\circ}$ & Pq Brasília & $28 \mathrm{~L}$ & 13.505 & 0,63 \\
\hline $41^{2}$ & Jd. Miriam/Pq Xangrilá & $22 \mathrm{~L}$ & 1.275 & 0,6 \\
\hline $42^{\circ}$ & VL Brandina & $33 \mathrm{~L}$ & 5.800 & 0,13 \\
\hline $43^{2}$ & NV Campos Eliseos/Sta/ Lucia & 470 & 57.491 & $-0,02$ \\
\hline $44^{3}$ & Jd. Esmeraldina/Jd. S. Pedro/Jd. D. & $62 \mathrm{~S}$ & 21.771 & $-0,07$ \\
\hline $45^{3}$ & São Quirino & $26 \mathrm{~L}$ & 22.567 & $-0,2$ \\
\hline $46^{3}$ & Fazendinha/Sta Bárbara & $12 \mathrm{~N}$ & 12.932 & $-0,2$ \\
\hline
\end{tabular}




\begin{tabular}{|c|c|c|c|c|}
\hline RANKING & BAIRRO & UTB SAR & POP & IQV \\
\hline $47^{\circ}$ & NV Mercedes & $65 \mathrm{~S}$ & 1.282 & $-0,56$ \\
\hline $48^{2}$ & Mauro Marcondes/O Verde & ++480 & 39.554 & $-1,02$ \\
\hline $49^{3}$ & S. Fernando/VL Orosimbo Maia/ & $58 \mathrm{~S}$ & 21.073 & $-1,28$ \\
\hline $50^{3}$ & Real Parque & $7 N$ & 3.487 & $-1,63$ \\
\hline 51. & Joaquim Egidio & $42 \mathrm{~L}$ & 1.738 & $-1,64$ \\
\hline 52 & $\mathrm{Pq}$ Valença & 450 & 26.669 & $-1,64$ \\
\hline $5^{3}$ & Jd. NV América & $66-A S$ & 2.772 & $-2,78$ \\
\hline $54^{3}$ & Jd. S. Cristovão & 500 & 10.717 & $-3,06$ \\
\hline $55^{3}$ & Icarai/Jd. das Bandeiras & $64 \mathrm{~S}$ & 12.985 & $-3,51$ \\
\hline $56^{3}$ & C. Gomes/Monte Belo/Chac. Gar & $21 \mathrm{~L}$ & 1.016 & $-3,87$ \\
\hline $57^{\circ}$ & Campo Grande/Florence & 460 & 18.273 & $-3,96$ \\
\hline $58^{2}$ & Sāo Marcos/Amaris & $10 N$ & 18.837 & $-4,69$ \\
\hline $59^{3}$ & Jd. Sāo Domingos/Campo Belo & $66 \mathrm{~S}$ & 5.311 & $-4,77$ \\
\hline $60^{3}$ & Carrefour/Galeria/FEAC & $29 \mathrm{~L}$ & 1.945 & $-5,83$ \\
\hline
\end{tabular}

Fonte: Observatório de Qualidade de Vida de Campinas, 1996.

TABELA 4: Posição das UTBs no ranking dos IQV "relativo"

RANKING

BAIRRO

UTB SAR

POP

IQV

Faz. Chapadão

Notre Dame/Alto Nova Campinas

Cidade Universitária

N. Campinas

PUCC/Pq das Universidades

VL Nova

Bairro das Palmeiras

Sāo Conrado

Castelo

NV Mercedes

$\mathrm{Pq}$ Imperador

Guanabara

$\mathrm{Pq}$ Jambeiro/Remonta

Jd. Fernanda

Mansões Sto Antonio/Sta. Cândida

Bosque

Primavera/Pq Taquaral

Jd. Miriam/Pq Xangrilá

Bonfim

Centro

S. Martin

C. Gomes/Monte Belo/Chac. Gar

Guará

$14 N$

$38 \mathrm{~L}$

876

1.251

7.469

7.072

$36 \mathrm{~L}$

$8 \mathrm{~N}$

958

6.010

1.520

38-A L

$39 \mathrm{~L}$

1.029

$18 \mathrm{~N}$

12.114

$65 \mathrm{~S}$

22-B L

1.282

1.289

$30 \mathrm{~L}$

11.560

1.613

$67 \mathrm{~S}$

2.143

3.285

$24 \mathrm{~L}$

14.645

8.539

1.275

8.623

16.626

2.380

1.016

3.744

1.738

Joaquim Egidio

Cambui

Ponte Preta

Pq. Via Norte

Centro Br. Geraldo

Real Parque

Jd. N. Sra. Auxiliadora

Jd. NV América

VL Costa e Silva/VL M.V.Cury

Carrefour/Galeria/FEAC

Jd. Aurélia

Maria Rosa

VL Brandina

NV. Europa/Pq da Figueira

Flamboyant

26.168

14.075

9.458

10.831

3.487

22.197

2.772

15.714

1.945

25.305

7.855

5.800

21.544

16.730

9,97

9,41

9,39

9,24

9,04

8,71

8,59

8,5

8,22

8,03

7,98

7,79

7,78

7,62

7,57

7,55

7,51

7,42

7,32

7,29

7,18

6,99

6,69

6,63

6,18

5,89

$32 \mathrm{~L}$ 


\begin{tabular}{|c|c|c|c|c|}
\hline RANKING & BAIRRO & UTB SAR & POP & IQV \\
\hline $40^{3}$ & Centro Sousas & $40 \mathrm{~L}$ & 8.219 & 2,86 \\
\hline $41^{3}$ & Chapadão & $17 \mathrm{~N}$ & 16.462 & 2,42 \\
\hline $42^{\circ}$ & DIC's/COHAB & 510 & 26.989 & 2,38 \\
\hline $43^{3}$ & V. Pompéia/Jd do Lago & $59 \mathrm{~S}$ & 21.995 & 2,12 \\
\hline $44^{3}$ & Jd. Proença & $57 \mathrm{~S}$ & 21.268 & 0,95 \\
\hline $45^{3}$ & NV Aparecida/Pe Anchieta & $11 \mathrm{~N}$ & 18.597 & 0,53 \\
\hline $46^{3}$ & Jd. São Domingos/Campo Belo & $66 \mathrm{~S}$ & 5.311 & 0,46 \\
\hline $47^{\circ}$ & Pq Brasilia & $28 \mathrm{~L}$ & 13.505 & 0,29 \\
\hline $48^{3}$ & Fazendinha/Sta Bárbara & $12 \mathrm{~N}$ & 12.932 & $-0,5$ \\
\hline $49^{3}$ & Jd. Esmeraldina/Jd. S.Pedro/Jd. & $62 \mathrm{~S}$ & 21.771 & $-1,56$ \\
\hline $50^{3}$ & Jd. S. Cristovão & 500 & 10.717 & $-1,78$ \\
\hline $5 t^{3}$ & Jd. dos Oliveiras/Swift & $61 S$ & 33.682 & $-1,88$ \\
\hline $52^{3}$ & Icarai/Jd das Bandeiras & $64 \mathrm{~S}$ & 12.985 & $-2,01$ \\
\hline $53^{3}$ & S. Fernando/NL Orosimbo Maia/ & $58 \mathrm{~S}$ & 21.073 & $-2,69$ \\
\hline $54^{2}$ & São Quirino & $26 \mathrm{~L}$ & 22.567 & $-2,87$ \\
\hline $55^{3}$ & $\mathrm{Pq}$ Valença & 450 & 26.669 & $-3,81$ \\
\hline $56^{3}$ & Campo Grande/Florence & 460 & 18.273 & $-4,63$ \\
\hline $57^{3}$ & São Marcos/Amarais & $10 \mathrm{~N}$ & 18.837 & $-5,04$ \\
\hline $58^{3}$ & Mauro Marcondes/O Verde & 480 & 39.554 & $-5,89$ \\
\hline $59^{\circ}$ & Jd. Garcia/Campos Eliseos & 440 & 59.354 & $-6,23$ \\
\hline $60^{\circ}$ & NV Campos Eliseos/Sta Lúcia & 470 & 57.491 & $-9,37$ \\
\hline
\end{tabular}

Fonte:Observatório de Qualidade de Vida de Campinas, 1996.

\section{GEOGRAFIAS DE SITUAÇÕES - PROBLEMA}

Viver em condições satisfatórias de qualidade de vida pressupõe ausências de "rupturas no fluxo da vida". Em outras palavras, a garantia de segurança para a vida das populações dos temitórios urbanos e rurais. Ao poder público e á comunidade cabem envidar esforços no sentido de cada vez mais minimizar os riscos a que estão submetidas. Se o I.Q.V. obtido para cada UTB informa sobre os determinantes sócio-sanitários sob as quais vivem os habitantes dessas UTBs, a análise de situações - problema que acometem esses habitantes aponta para os riscos diferenciados entre os distintos espaços territoriais.

Quatro situações problema foram consideradas, a princípio, e examinadas pelo grupoexecutivo: saúde, transporte, segurança-pública e meio ambiente..

As duas primeiras foram as que apresentaram dados passiveis de desagregaçãotemitorial entre as 4 grandes regiões da cidade de Campinas, não propiciando, infelizmente, possibilidades de desagregação para as 60 UTBs. A análise das 4 regiões pode ser obtida junto à P.M. de Campinas ou com os autores. 


\section{OBSERVAÇÕES FINAIS}

O ritmo de crescimento das cidades brasileiras vai muito além da sua capacidade de produzir dados atualizados que demonstrem esse crescimento, e apontem com clareza as conseqüências sobre a qualidade de vida das populações urbanas. Se por um lado, há tradição de monitoramento contínuo, em intervalos anuais, mensais e até diários, dos indicadores econômicos, como por exemplo, a inflação e o comércio exterior, o mesmo não se pode dizer dos dados sociais, que possuem os censos decenais como um dos seus principais termômetros.

Por isso, falar da qualidade de vida em Campinas como um todo, para o ano de 1996, utilizando-se de dados coletados em 1991, parece um exercício anacrônico, quando há dados mais recentes dizendo por exemplo, que nos últimos 5 anos Campinas ganhou 31 novas favelas (Folha de São Paulo, 1996)

O conceito de qualidade de vida é bastante abrangente e sua representação através de dados quantitativos pressupõe uma gama variada de indicadores. Entretanto, as bases de dados tradicionais existentes nos municípios brasileiros não possuem amplo leque de indicadores dequalidade de vida urbana desagregados por pequenos temitórios. Esta deficiência cria lacunas na totalidade da apresentação quantitativa do fenômeno em pequenos espaços locais, no caso de Campinas, nas UTBs, formando "gavetas vazias" nas bases de dados municipais, que precisam ser "preenchidas", para darem conta da descrição local da qualidade de vida.

Entretanto, tal exercício lança bases importantes para consolidar um processo intersetorial de monitoramento contínuo da qualidade de vida na cidade, ao equacionar importantes questões no processo de explorar as bases de dados atualmente existentes no município, tais como: 0 desenvolvimento de uma percepção clara da necessidade de base cartográfica digitalizada para toda a cidade; a criação de elemențos que contribuam para o desenvolvimento de uma "cultura do local", ao priorizar na análise a menor unidade territorial do município, a UTB; estreitamento das relações entre secretarias mais tradicionais e outras recentemente criadas, como por exemplo, a Secretaria de Segurança Pública; reconhecimento e valorização de bancos de dados pouco conhecidos e pouco explorados, como por exemplo, o da Defesa Civil Municipal; reconhecimento concreto da necessidade de criação de um sistema municipal de informações de meioambiente, para que se expanda o conceito de qualidade de vida.

Mais ainda, o presente exercício de elaboração do indicador de qualidade sócio-sanitária de Campinas propiciou o levantamento da discussão dos dois critérios "absoluto" e "relativo" 
como elementos importantes na determinação de caminhos alternativos e/ou complementares para a formulação de políticas públicas.

A construção desse indicador, e o conseqüente desenvolvimento do "Observatório", poderia ainda favorecer Campinas como uma fonte importante de subsídios para outras localidades que façam parte do movimento das cidades saudáveis, garantindo à Campinas local privilegiado no ranking das cidades saudáveis.

Pode-se ainda, almejar para o futuro, que a construção de um indicador de qualidade de vida urbana e o seu conseqüente monitoramento ao longo do tempo, pode redundar na "invenção" de um termômetro eficiente que possa medir, de forma indireta, a temperatura da gestão pública, atualmente tão carente de mecanismos avaliativos eficazes. Nesse sentido, os servidores públicos podem se colocar, ao mesmo tempo, como agentes de aperfeiçoamento da qualidade de vida da sua cidade, e também como beneficiários dessa melhoria da qualidade de vida no seu próprio ambiente urbano.

O lançamento, nessa publicação, da idéia de criação da Rede de Observação da Qualidade de Vida em Campinas visa criar as bases para a construção de uma nova visão de cidade, uma cidade para todos, que seja economicamente eficiente, socialmente equânime e sustentável do ponto de vista do meio ambiente. Para alcançar esse intento, há necessidade de, cada vez mais, estreitar-se relações e parcerias entre governo, setor privado e sociedade civil.

\section{REFERÊNCIAS BIBLIOGRÁFICAS}

CARSTAIRS, V. \& MORRIS, R. Deprivation and mortality: an altemative to social class? Community Med., 11:210-19, 1989a.

CARSTAIRS, V. \& MORRIS, R. Deprivation: explaining differences in mortality between Scotland, England and Wales. BMJ, 299: 886-89, $1989 \mathrm{~b}$.

DUHL, L.. Conditions for healthy cities, diversity, game boards and social entrepreneurs. Environ. Urban., 5:112-24, 1993.

FUNDAÇÃO IBGE Censo demográfico do Brasil. Rio de Janeiro, 1991.

LOCKER, D. Measuring social inequality in dental health services research: individual, household and area-based measures. Toronto, Department of Community Dentistry and Community Dental Health Services Research Unit, Faculty of Dentistry, University of Toronto, 1992.

PARETO, V.E. A model to assess urban conditions and dimension development projects. Habitat 
Int., 16(: 99-117, 1992.

PNUD Informe sobre desarrollo humano 1993. Madrid, CIDEAL., 1993.

PREFEITURAMUNICIPALDECAMPINAS Construindo novos rumos para a administração pública. Campinas, Prefeitura Municipal de Campinas, 1996.

PREFEITURAMUNICIPALDECAMPINAS Campinas: planodiretor. Campinas, Prefeitura Municipal de Campinas, 1995.

SPOSATI, A., KOGA, D., AKERMAN, M, MARQUES, M.B Mapa da exclusão/inclusão social da cidade de São Paulo. São Paulo, 1996.

WERNA, E. United Nations Agencies' Urban Policies and Health. [Paper presented to the Conference 'Urban Health Research: Implications for Policy'. London School of Hygiene and Tropical Medicine, London, 1994.]

WERNA, E. The evaluation of healthy city projects in developing countries. Habitat Int., 19(3):1-13, 1995.

Summary: Given the assumption that the construction of a Healthy City demands a joint effort of all its inhabitants and that this is possible from a scientific basis the authors of the text present the experience of the Campinas Quality of Life Observatory Project that aims at the design of a quality of life index instrument. The phases described are the first intersectorial mobilization, conceptualization and localization of administrative and planning areas with special basis for the urbanistic project to be developed by future regional scope administrations. Second, the construction of the quality of life index itself from the 1991 demographic database and next discussions and recommendations. Such exercise has laid the bases for the consolidation of an efficient and feasible tool of continuous monitoring of an intersectorial process of quality of life betterment such as the one proposed by WHO for Healthy Cities.

Key words: construction; healthy city; quality of life

Agradecimentos:

Agradeço a colaboração competente de Adriana Pires na edição do texto. $E$ as profas Ausonia Donato, Maria Erlinda Duckur Cassab e Helder Leal da Costa por suporte instituciona; e intelectual ao trabalho. 